\title{
Extension of Kirk-Saliga Fixed Point Theorem in a Metric Space with a Reflexive Digraph
}

\author{
Karim Chaira, ${ }^{1}$ Abderrahim Eladraoui $\mathbb{D}^{1},{ }^{2}$ Mustapha Kabil, ${ }^{2}$ and Samih Lazaiz ${ }^{1}$ \\ ${ }^{1}$ Laboratory of Algebra, Analysis and Applications, Faculty of Sciences Ben M'sik, University of Hassan II Casablanca, \\ Casablanca, Morocco \\ ${ }^{2}$ Laboratory of Mathematics and Applications, Faculty of Sciences and Technologies, Mohammedia, \\ University of Hassan II Casablanca, Casablanca, Morocco \\ Correspondence should be addressed to Abderrahim Eladraoui; a.adraoui@live.fr
}

Received 4 October 2017; Accepted 31 December 2017; Published 1 February 2018

Academic Editor: Nawab Hussain

Copyright (C) 2018 Karim Chaira et al. This is an open access article distributed under the Creative Commons Attribution License, which permits unrestricted use, distribution, and reproduction in any medium, provided the original work is properly cited.

We extend the result of Kirk-Saliga and we generalize Alfuraidan and Khamsi theorem for reflexive graphs. As a consequence, we obtain the ordered version of Caristi's fixed point theorem. Some concrete examples are given to support the obtained results.

\section{Introduction}

Fixed point theory is one of the most useful tools in mathematics; it is used to solve many existence problems such as differential equations, control theory, optimization, and several other branches (for the literature see [1]). The most well-known fixed point result is Banach contraction principle [2]; it is famous for its applications, proving the existence of solution of integral equations by converting the problem to fixed point problem (see [3]). Recall that a point $x \in X$ is called a fixed point for a map $T$ : $X \rightarrow X$ if $T x=x$. Due to its importance, this theorem found a number of generalizations and extensions in many directions; for more details see [4] and the references therein. In 1976, Caristi (see [5]) gave an elegant generalization of Banach contraction principle, where the assumption that " $T: X \rightarrow X$ is continuous" is dropped and replaced by a weak assumption. Since then, various proofs, extensions, and generalizations are given by many authors (see [6-8]). It is worth mentioning that Caristi's fixed point theorem is equivalent to the Ekeland variational principle [8]. Also, it characterizes the completeness of the metric space as showed by Kirk in [9]. Among those generalizations, there is KirkSaliga fixed point theorem (see [10]) which states that any map $T: X \rightarrow X$ has a fixed point provided that $X$ is complete metric space and there exist an integer $p \in \mathbb{N}$ and a lower semicontinuous function $\varphi: X \rightarrow[0, \infty)$ such that

$$
d(x, T x) \leq \varphi(x)-\varphi\left(T^{p} x\right)
$$

and $\varphi(T x) \leq \varphi(x)$ for any $x \in X$. For more on the latter result, one can consult [11].

Recently, Ran and Reurings [12] extend the Banach contraction principle in the context of partially ordered set where the contraction is restricted to the comparable elements which allowed them to give a meaningful application to linear and nonlinear matrix equations. Moreover, Nieto and Rodríguez-López in [13] have weakened the continuity assumption using a more suitable condition where the order is combined with the topological properties. For more details, one can consult $[14,15]$. Also, in [16] Alfuraidan and Khamsi gave an analogue version of Caristi's fixed point theorem in the setting of partially ordered metric space where the inequality holds only for comparable elements. However, the new approach in their work is mixing the concept of the reflexive acyclic digraph with fixed point results. In this article, we discuss an extension of Kirk-Saliga result and we generalize Alfuraidan and Khamsi theorem for reflexive graphs. As a corollary, we obtain the ordered version of Caristi's fixed point theorem. Some concrete examples are given to support the obtained results. Throughout this paper we denote by $\mathbb{N}$ the set of all integers and by $\mathbb{N}^{*}$ the set of all positive integers. 


\section{Preliminaries}

We start by recalling some basic notions on graphs borrowed from [17].

Definition 1. Let $V$ be an arbitrary set.

(i) A directed graph, or digraph, is a pair $G=(V, E)$, where $E$ is a subset of the Cartesian product $V \times V$. The elements of $V$ are called vertices or nodes of $G$ and the elements of $E$ are the edges also called oriented edges or arcs of $G$. An edge of the form $(v, v)$ is a loop on $v$. Another way to express that $E$ is a subset of $V \times V$ is to say that $E$ is a binary relation over $V$. Given a digraph $G$, the set of vertices (of edges) of $G$ is denoted by $V(G)$ $(E(G))$.

(ii) The digraph $G=(V, E)$ is said to be transitive if whenever $(x, y) \in E$ and $(y, z) \in E,(x, z) \in E$.

Definition 2. A digraph $G=(V, E)$ is said to be reflexive if $\Delta:=\{(v, v) \mid v \in V\}$ is a subset of $E$. Otherwise, every vertex has a loop.

Definition 3. Let $G=(V, E)$ be a digraph.

(i) A vertex $x$ is said to be isolated if for all vertex $y \neq x$, we have neither $(x, y) \in E$ nor $(y, x) \in E$.

(ii) Two vertices $x, y \in V$. A path in $G$, from (or joining) $x$ to $y$, is a sequence of vertices $p=\left\{a_{i}\right\}_{0 \leq i \leq n}, n \in \mathbb{N}^{*}$ such that $a_{0}=x, a_{n}=y$ and $\left(a_{i}, a_{i+1}\right) \in E$, for all $i \in\{0,1, \ldots, n-1\}$. The integer $n$ is the length of the path $p$. If $x=y$ and $n>1$, the path $p$ is called a directed cycle. An acyclic digraph is a digraph which has no directed cycle.

(iii) We denote by $y \in[x]_{G}$ the fact that $y$ can be reached from $x$ by means of a path in $G$.

A metric space $(X, d)$ endowed with a digraph $G$ such that $V(G)=X$ is denoted by $(X, d, G)$. The following notion of regularity is borrowed from Alfuraidan and Khamsi in [16] that considered it for posets.

Definition 4 . Let $(X, d, \preceq)$ be a partially ordered metric space. We say that $X$ satisfies the condition (OSC) if for any decreasing sequence $\left\{x_{n}\right\} \subseteq X$ that is convergent to $x \in X$, $x=\inf \left\{x_{n}: n \in \mathbb{N}\right\}$.

In the setting of digraphs, the analogue of the infimum of chain may be stated as follows.

Definition 5. Let $(X, d, G)$ be metric space endowed with a digraph. We say that $X$ satisfies the condition (OSCL) if for any sequence $\left\{x_{n}\right\} \subseteq X$ that is convergent to $x \in X$ and for all $n \in \mathbb{N}, x_{n+1} \in\left[x_{n}\right]_{G}, x \in\left[x_{n}\right]_{G}$ for all $n \in \mathbb{N}$ and if there exists $y \in X$ such that $y \in\left[x_{n}\right]_{G}$, for all $n \in \mathbb{N}$, then $y \in[x]_{G}$.
Remark 6. Let $(X, d, \preceq)$ be a partially ordered metric space. Let $G_{\preceq}$ be the digraph associated with the order $\preceq$ (see [16]). One can see that

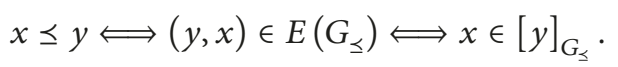

Under the above observations, the (OSCL) property is reduced to the (OSC) condition.

Let $\omega$ be the first transfinite ordinal and let $\Omega$ be the first uncountable transfinite ordinal. $\omega$ is the order type of $\mathbb{N}$ "the set of integers" and $\Omega$ is the order type of $\mathbb{R}$ the set of real numbers. Note that, for each $\xi<\Omega$, $\xi$ is countable.

Proposition 7 (see [11]). The following is valid:

(i) The ordinal $\Omega$ cannot be attained via sequential limits of countable ordinals. That is if $\left\{\alpha_{n}\right\}$ is an ascending sequence of countable ordinals, then the ordinal

$$
\alpha=\sup \left\{\alpha_{n}\right\}=\lim \alpha_{n}
$$

is countable too.

(ii) Each second kind countable ordinal is attainable via such sequences. In other words: if $\alpha<\Omega$ is of second kind (ordinal limit), then there exists a strictly ascending sequence $\left\{\alpha_{n}\right\}$ of countable ordinals with property (3).

The following result is needed throughout this work; for the proof see [18, Proposition A.6, pp. 284].

Proposition 8. Suppose that a sequence $\left\{x_{\alpha}\right\}_{\alpha \in \Omega} \subseteq \mathbb{R}$ is bounded and either nonincreasing or nondecreasing. Then there exists $\beta \in \Omega$ such that $x_{\alpha}=x_{\beta}$ for all $\Omega>\alpha \geq \beta$.

We conclude this section by the following useful definitions.

Definition 9. Let $(X, d, G)$ be metric space endowed with a digraph, $p \in \mathbb{N}$ and $\varphi: X \rightarrow[0,+\infty$ [ a lower semicontinuous function. Let $T: X \rightarrow X$ be a self-mapping. We say the following:

(1) $T$ is a $G$-monotone if for all $(x, y) \in X^{2}$,

$$
x \in[y]_{G} \Longrightarrow T x \in[T y]_{G} .
$$

(2) $T$ is a $G$-Caristi mapping if for all $x \in X$,

$$
T x \in[x]_{G} \Longrightarrow d(T x, x) \leq \varphi(x)-\varphi(T x) .
$$

(3) $T$ is a $G$-Kirk-Saliga mapping if for all $x \in X$,

$T x \in[x]_{G} \Longrightarrow\left\{\begin{array}{l}(\mathrm{KS} 1): d(T x, x) \leq \varphi(x)-\varphi\left(T^{p} x\right) \\ (\mathrm{KS} 2): \varphi(T x) \leq \varphi(x) .\end{array}\right.$ 


\section{Main Results}

Theorem 10. Let $(X, d, G)$ be a complete metric space endowed with a reflexive digraph satisfying the (OSCL) condition. Let $T: X \rightarrow X$ be a G-monotone and G-Kirk-Saliga mapping. If there exists an element $x_{0} \in X$ such that $T x_{0} \in\left[x_{0}\right]_{G}$, then $T$ admits a fixed point in $X$.

Proof. If $p=0$ then $T x=x$, for all $x \in X$ such that $T x \in[x]_{G}$. Assume that $p \geq 1$ and consider the function $\phi$ defined from $X$ into $[0,+\infty[$ by

$$
\phi(x)=\sum_{i=0}^{p-1} \varphi\left(T^{i} x\right), \quad \forall x \in X .
$$

The idea of the proof is to construct a transfinite orbit $\left(x_{\alpha}\right)_{\alpha \in \Omega}$, where $\Omega$ is the first uncountable ordinal satisfying, for each $\alpha \in \Omega$,

$$
\begin{aligned}
& A(\alpha): T x_{\alpha}=x_{\alpha+1} ; \\
& B(\alpha): x_{\alpha}=\lim _{\lambda \rightarrow \alpha^{-}} x_{\lambda}, \text { whenever } \alpha \text { is an ordinal limit; } \\
& C(\alpha): x_{\alpha} \in\left[x_{\mu}\right]_{G} \text {, whenever } \mu \prec \alpha ; \\
& D(\alpha): d\left(x_{\alpha}, x_{\mu}\right) \leq \phi\left(x_{\mu}\right)-\phi\left(x_{\alpha}\right), \text { whenever } \mu \preceq \alpha .
\end{aligned}
$$

Consider the sequence $\left\{x_{n}\right\}$ defined for each $n \in \mathbb{N}$ by $x_{n+1}=T x_{n}$. Since $T x_{0} \in\left[x_{0}\right]_{G}$ and using the monotony of $T$, we obtain $x_{n+1} \in\left[x_{n}\right]_{G}$ for each $n \in \mathbb{N}$. According to (KS2), the nonnegative sequence $\left\{\phi\left(x_{n}\right)\right\}$ is decreasing and then converges. From (KS1), we get that for all integers $n>m$

$$
\begin{aligned}
d\left(x_{m}, x_{n}\right) & \leq \sum_{i=m}^{n-1} d\left(x_{i}, x_{i+1}\right) \leq \sum_{i=m}^{n-1} \phi\left(x_{i}\right)-\phi\left(x_{i+1}\right) \\
& \leq \phi\left(x_{m}\right)-\phi\left(x_{n}\right)
\end{aligned}
$$

Hence, $\left\{x_{n}\right\}$ is a Cauchy sequence and then converges to $x_{\omega} \in$ $X$. Let us put $x_{\omega+1}=T x_{\omega}$. Clearly the properties $A(\alpha)-D(\alpha)$ are satisfied for each $\alpha \leq \omega$. Let $\beta \in \Omega$. Assume that the orbit $\left\{x_{\alpha}\right\}_{\alpha<\beta}$ has been defined. We need to define $x_{\beta}$ and show that the four properties $A(\beta)-D(\beta)$ hold. For that, we have to distinguish two cases, when $\beta$ is an immediate successor or $\beta$ is an ordinal limit. Clearly $A(\beta)$ and $B(\beta)$ are satisfied; let us focus on $C(\beta)$ and $D(\beta)$.

\section{Claim 1 ( $C(\beta)$ holds)}

Case 1. Assume that $\beta$ is an ordinal limit; that is, there exists a strictly ascending sequence $\left(\beta_{n}\right)_{n}$ of ordinals in $\Omega$ such that $\beta=\sup \left\{\beta_{n} ; n \in \mathbb{N}\right\}$ and $\beta_{m} \preceq \beta_{n} \prec \beta$ whenever $m \leq n$. Since $D(\alpha)$ holds for all $\alpha \prec \beta$, we get

$$
d\left(x_{\beta_{n}}, x_{\beta_{m}}\right) \leq \phi\left(x_{\beta_{m}}\right)-\phi\left(x_{\beta_{n}}\right),
$$

which implies that $\left(\phi\left(x_{\beta_{n}}\right)\right)_{n}$ is decreasing sequence in $[0, \infty)$ and hence it is convergent. Then $\left(x_{\beta_{n}}\right)$ is Cauchy sequence, so it converges in $X$. Set $x_{\beta}=\lim _{n \rightarrow \infty} x_{\beta_{n}}$. By (OSCL) property, we obtain $x_{\beta} \in\left[x_{\beta_{n}}\right]_{G}$ for all $n \in \mathbb{N}$. Let $\alpha \prec \beta$. There exists $n_{0} \in \mathbb{N}$ such that for each $n \geq n_{0}$ we have

$$
\alpha \preceq \beta_{n} \prec \beta,
$$

and thus for each $n \geq n_{0}$,

$$
x_{\beta_{n}} \in\left[x_{\alpha}\right]_{G}, x_{\beta} \in\left[x_{\beta_{n}}\right]_{G} \Longrightarrow x_{\beta} \in\left[x_{\alpha}\right]_{G} .
$$

Since $\alpha$ is taken arbitrary, we obtain $C(\beta)$.

Case 2. Assume that $\beta$ is an immediate successor; there exists $\alpha \prec \beta$ such that $\beta=\alpha+1$.

(i) If $\alpha$ is an immediate successor, there exists an ordinal $\mu$ such $\alpha=\mu+1$. From $C(\alpha)$, we have $x_{\alpha} \in\left[x_{\mu}\right]_{G}$ and using the $G$-monotonicy of $T$ it follows that $x_{\beta} \in$ $\left[x_{\alpha}\right]_{G}$ and so $C(\beta)$ holds.

(ii) If $\alpha$ is an ordinal limit, from Proposition 7 , there exists an ascending sequence $\left\{\alpha_{n}\right\} \subset \Omega$ such that $\alpha=\sup \left\{\alpha_{n}: n \in \mathbb{N}\right\}$. From $B(\alpha)$ we have $x_{\alpha}=$ $\lim _{n \rightarrow+\infty} x_{\alpha_{n}}$. Using the (OSCL) condition, we have $x_{\alpha} \in\left[x_{\alpha_{n}}\right]_{G}$. Since $T$ is $G$-monotone, $x_{\beta} \in\left[x_{\alpha_{n}+1}\right]_{G}$ and as $x_{\alpha_{n}+1} \in\left[x_{\alpha_{n}}\right]_{G}$, we get $x_{\beta} \in\left[x_{\alpha_{n}}\right]_{G}$. Again, (OSCL) insures that $x_{\beta} \in\left[x_{\alpha}\right]_{G}$. Then $C(\beta)$ holds.

Claim $2(D(\beta)$ holds $)$

Case 1. Assume that $\beta$ is ordinal limit. Let $\alpha \prec \beta$. There exists $n_{0} \in \mathbb{N}$ such that for each $n \geq n_{0}$ we have

$$
\alpha \preceq \beta_{n} \prec \beta \text {. }
$$

Then we get for each $n \geq n_{0}$ that

$$
d\left(x_{\alpha}, x_{\beta_{n}}\right) \leq \phi\left(x_{\alpha}\right)-\phi\left(x_{\beta_{n}}\right),
$$

and for all $i \in\{0,1, \ldots, p-1\}$

$$
\lim _{n \rightarrow \infty} T^{i} x_{\beta_{n}}=\lim _{n \rightarrow \infty} x_{\beta_{n}+i}=x_{\beta} .
$$

Since $\varphi$ is lower semicontinuous, we get

$$
\varphi\left(x_{\beta}\right) \leq \liminf _{n \rightarrow \infty} \varphi\left(T^{i} x_{\beta_{n}}\right) .
$$

From $C(\beta)$, we have $x_{\beta} \in\left[x_{\beta_{n}}\right]_{G}$ for all $n \in \mathbb{N}$. Using the same argument as above, we get $T x_{\beta} \in\left[x_{\beta_{n}}\right]_{G}$ and (OSCL) insures that $T x_{\beta} \in\left[x_{\beta}\right]_{G}$. Hence, for all $i \in\{0,1, \ldots, p-1\}$, $T^{i+1} x_{\beta} \in\left[T^{i} x_{\beta}\right]_{G}$. This implies that

$$
\varphi\left(T^{p} x_{\beta}\right) \leq \varphi\left(T^{p-1} x_{\beta}\right) \leq \cdots \leq \varphi\left(T x_{\beta}\right) \leq \varphi\left(x_{\beta}\right) .
$$

By passing to limit superior in inequality (13), it follows that

$$
\begin{aligned}
d\left(x_{\alpha}, x_{\beta}\right) & \leq \phi\left(x_{\alpha}\right)-\liminf _{n \rightarrow+\infty} \phi\left(x_{\beta_{n}}\right) \\
& \leq \phi\left(x_{\alpha}\right)-\sum_{i=0}^{p-1} \liminf _{n \rightarrow+\infty} \varphi\left(T^{i} x_{\beta_{n}}\right) \\
& \leq \phi\left(x_{\alpha}\right)-p \varphi\left(x_{\beta}\right) \leq \phi\left(x_{\alpha}\right)-\phi\left(x_{\beta}\right) .
\end{aligned}
$$

Hence, $D(\beta)$ holds.

Case 2. Assume that $\beta=\alpha+1$ is an immediate successor; we have shown above that $C(\beta)$ holds. Then $T x_{\alpha}=x_{\beta} \in\left[x_{\alpha}\right]_{G}$ and by assumption we get

$$
d\left(x_{\alpha}, x_{\beta}\right) \leq \phi\left(x_{\alpha}\right)-\phi\left(x_{\beta}\right),
$$


and for all $\gamma \preceq \alpha$, we have

$$
d\left(x_{\gamma}, x_{\alpha}\right) \leq \phi\left(x_{\gamma}\right)-\phi\left(x_{\alpha}\right) \text {. }
$$

The triangle inequality implies that

$$
d\left(x_{\gamma}, x_{\beta}\right) \leq \phi\left(x_{\gamma}\right)-\phi\left(x_{\beta}\right),
$$

for each $\gamma \preceq \beta$, which completes the proof of $D(\beta)$ in both cases.

Thus, the orbit $\left(x_{\alpha}\right)_{\alpha \in \Omega}$ is well constructed. Since $\left\{\phi\left(x_{\alpha}\right)\right\}$ is nonincreasing on $\left\{x_{\alpha}\right\}$ and $\Omega$ is uncountable, there must exist $\alpha_{0} \in \Omega$ such that $\phi\left(x_{\alpha}\right)$ is constant for all $\alpha \geq \alpha_{0}$. From $D\left(\alpha_{0}+1\right)$, we get

$$
d\left(x_{\alpha_{0}+1}, x_{\alpha_{0}}\right) \leq \phi\left(x_{\alpha_{0}}\right)-\phi\left(x_{\alpha_{0}+1}\right)=0 .
$$

Hence, $T x_{\alpha_{0}}=x_{\alpha_{0}+1}=x_{\alpha_{0}}$.

We support our result by giving an example of a mapping which is $G$-Kirk-Saliga mapping, for some integer $p>1$, but not $G$-Caristi.

Example 11. Consider the metric space $(X, d)$, where $X=$ $[0,1]$ and $d(x, y)=|x-y|$, for all $x, y \in X$. Endow $X$ with the directed graph $G=(X, E)$ represented in Figure 1, where

$$
\begin{aligned}
E & =\Delta \cup\left\{\left(\sqrt{\frac{1}{2}}, 0\right),(1,0),\left(\frac{1}{2^{n}}, 0\right),\left(\frac{1}{2^{n}}, \frac{1}{2^{n+1}}\right): n\right. \\
& \left.\in \mathbb{N}^{*}\right\} .
\end{aligned}
$$

Consider the function $\varphi: X \rightarrow[0,+\infty[$ defined by

$$
\varphi(x)= \begin{cases}\sqrt{x}, & \text { if } x \in[0,1[; \\ 0, & \text { if } x=1,\end{cases}
$$

and the mapping $T: X \rightarrow X$ defined by $T x=x^{2}$, if $x \in[0,1[$; $T 1=\sqrt{1 / 2}$.

One can see that $T 1 \notin[1]_{G}, T \sqrt{1 / 2} \notin[\sqrt{1 / 2}]_{G}$ and

$$
X_{G}:=\left\{x \in X: T x \in[x]_{G}\right\}=\left\{0, \frac{1}{2^{n}}: n \in \mathbb{N}^{*}\right\} .
$$

We verify the following assertions:

(i) $(X, d)$ is complete and $T$ is $G$-monotone obviously.

(ii) $G$ satisfies the (OSCL) property. Indeed, let $\left\{x_{n}\right\}$ be a sequence in $X$ such that $\left\{x_{n}\right\}$ converges to some $x \in X$ and $x_{n+1} \in\left[x_{n}\right]_{G}$, for all $n \in \mathbb{N}$. Two cases to distinguish are as follows:

(1) There exists $n_{0} \in \mathbb{N}$ such that $x_{n}=x_{n_{0}}$, for all $n \geq$ $n_{0}$. Then for all $n \geq n_{0}, x_{n}=x$. If $x$ is an isolated vertex, the (OSCL) is obviously satisfied. If not, $y \in\left[x_{n}\right]_{G}$ for all $n \in \mathbb{N}$ implies $y \in[x]_{G}$. Thus, (OSCL) is satisfied.

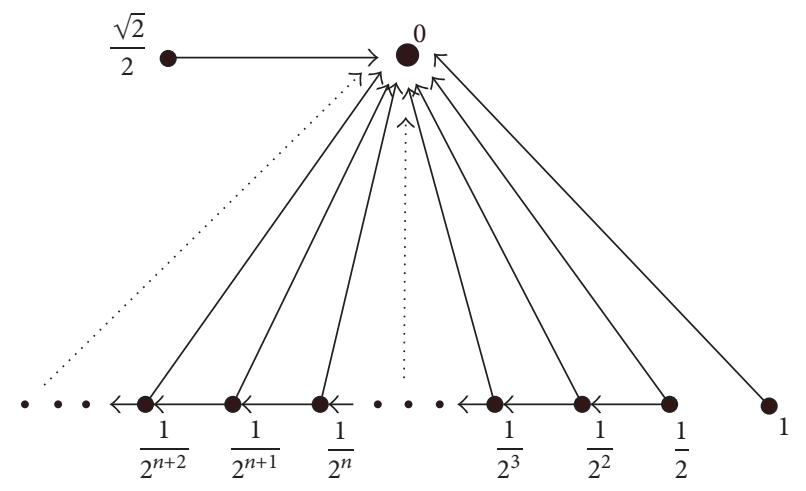

FIgURE 1: Graph $G$ (the loops and the isolated vertices are not represented).

(2) For all $k \in \mathbb{N}$, there exists $m_{k} \in \mathbb{N}$ such $x_{m_{k}} \neq x_{k}$. Then $\left\{x_{n}\right\} \subseteq\left\{1 / 2^{n}: n \in \mathbb{N}\right\}$; that is, there exists a nondecreasing function $\psi: \mathbb{N} \rightarrow \mathbb{N}$ such that $x_{n}=1 / 2^{\psi(n)}$ for all $n \in \mathbb{N}$, and $x=0$. If $y \in$ $\left[x_{n}\right]_{G}$ for all $n \in \mathbb{N}$, then $y=0$. Thus, (OSCL) is satisfied.

(iii) $T$ is $G$-kirk-Saliga mapping in $X$ with $p=3$. Indeed, for all $x \in X_{G}$,

$$
\begin{gathered}
x-x^{2} \leq \sqrt{x}-x^{4} \Longleftrightarrow \\
d(x, T x) \leq \varphi(x)-\varphi\left(T^{3} x\right),
\end{gathered}
$$

but $T$ is not $G$-Caristi mapping, since

$$
d\left(\frac{1}{2}, T \frac{1}{2}\right)>\varphi\left(\frac{1}{2}\right)-\varphi\left(T \frac{1}{2}\right) .
$$

(iv) $T 0 \in[0]_{G}$.

and $T$ admits a fixed point in $X$ which is 0 .

If we remove the (OSCL) property, we are not certain that the fixed point will be obtained. Let us illustrate that by this counterexample.

Example 12. Replace in the above example the digraph $G$ by the digraph $G^{\prime}$ represented in Figure 2, where

$$
E^{\prime}=\Delta \cup\left\{\left(\frac{1}{2^{n}}, 0\right),\left(\frac{1}{2^{n}}, 1\right),\left(\frac{1}{2^{n}}, \frac{1}{2^{n+1}}\right): n \in \mathbb{N}^{*}\right\},
$$

and we consider the mapping $T: X \rightarrow X$ defined as follows:

$$
\begin{aligned}
& T 0=1 ; \\
& \left.T x=x^{2}, \quad \text { if } x \in\right] 0,1[; \\
& T 1=0 .
\end{aligned}
$$

One can see that $G^{\prime}$ satisfies (OSC) property but does not satisfy the (OSCL), since $1 / 2^{n} \rightarrow 0$ and for all $n \in \mathbb{N}$, $0 \in\left[1 / 2^{n}\right]_{G^{\prime}}$ and $1 \in\left[1 / 2^{n}\right]_{G^{\prime}}$ but $1 \notin[0]_{G^{\prime}}$. The mapping $T$ satisfies all others conditions of Theorem 10 but has no fixed point in $X$. 


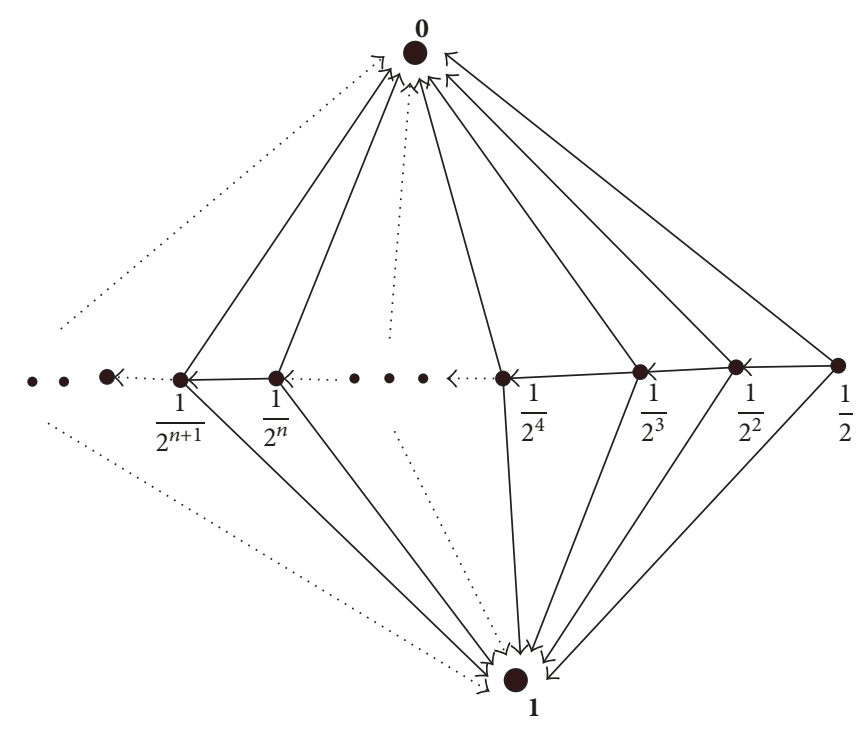

FIGURE 2: Graph $G^{\prime}$ (the loops and the isolated vertices are not represented).

Corollary 13. Let $(X, d, G)$ be a complete metric space endowed with a reflexive digraph which satisfies the (OSCL) property. Let $n=\prod_{i=0}^{k} p_{i}^{\alpha_{i}}$, where $p_{i}$ is prime integer and $\left(k, \alpha_{i}\right) \in \mathbb{N} \times \mathbb{N}^{*}$, for each $i \in\{0,1, \ldots, k\}$, and let $T: X \rightarrow X$ be a G-monotone mapping such that there exists $j \in\{0,1, \ldots, k\}$, for all $x \in X$,

$$
T^{p_{j}} x \in[x]_{G} \Longrightarrow\left\{\begin{array}{l}
\max \left\{d\left(x, T^{p_{i}} x\right): i \in\{0,1, \ldots, k\}\right\} \leq \varphi(x)-\varphi\left(T^{n} x\right), \\
\varphi\left(T^{p_{j}} x\right) \leq \varphi(x) .
\end{array}\right.
$$

Then $T$ admits a fixed point in $X$ provided that there exists an element $x_{0} \in X$ such that $T x_{0} \in\left[x_{0}\right]_{G}$.

Proof. Clearly, $T^{p_{j}}$ satisfies all conditions of Theorem 10; then there exists $\bar{x} \in X$ such that $T^{p_{j}} \bar{x}=\bar{x}$ and so, $T^{n} \bar{x}=\bar{x}$. Since $T^{p_{j}} \bar{x} \in[\bar{x}]_{G}$, we get from (29) that

$$
\begin{aligned}
d\left(\bar{x}, T^{p_{i}} \bar{x}\right) \leq \varphi(\bar{x})-\varphi\left(T^{n} \bar{x}\right)= & 0, \\
& \forall i \in\{0,1, \ldots, k\} \backslash\{j\} .
\end{aligned}
$$

Now, let $i \in\{0,1, \ldots, k\} \backslash\{j\}$; then $T^{p_{i}} \bar{x}=\bar{x}$. By Bezout identity, there exists $(u, v) \in \mathbb{Z}^{2}$ such that $u v \leq 0$ and $p_{j} u+p_{i} v=1$. Without loss of generality, we suppose that $u<0$ and $v>0$. Since $T^{p_{j}} \bar{x}=\bar{x}$ and $T^{p_{i}} \bar{x}=\bar{x}$, then $T^{1-p_{j} u} \bar{x}=T \bar{x}$ and $T^{p_{i} v} \bar{x}=\bar{x}$. Since $p_{i} v=1-p_{j} u$, then $T \bar{x}=\bar{x}$.

We conclude this work by a discussion about preordered sets.

Let $(X, \leqslant)$ be a preordered set; that is, the binary relation " $\leqslant$ " is reflexive and transitive.

Given a reflexive digraph $G=(X, E)$, we can always define a preorder $\leqslant_{G}$ on $X$ as follows:

$$
x \leqslant_{G} y \Longleftrightarrow x \in[y]_{G} .
$$

Conversely, if $(X, \leqslant)$ is a preordered set, we define the reflexive $\operatorname{digraph} G_{\leqslant}$as follows: two vertices $x, y \in X$ are connected by an $\operatorname{arc}$ from $x$ to $y$ if $x \leqslant y$. Note that $G_{\leqslant}$is transitive (i.e., if $(x, y) \in E\left(G_{\leqslant}\right)$and $(y, z) \in E\left(G_{\leqslant}\right)$; then $\left.(x, z) \in E\left(G_{\leqslant}\right)\right)$, so $x \in[y]_{G_{\leqslant}} \Leftrightarrow(x, y) \in E\left(G_{\leqslant}\right)$. These remarks lead to the following definition.

Definition 14 . Let $(X, \leqslant)$ be a preordered set. We say that $(X, \leqslant$ ) satisfies the (OSCL) condition if and only if $G_{\leqslant}$satisfies the (OSCL) condition.

We shall say that $T: X \rightarrow X$ is $\leqslant-$ monotone (resp., $\leqslant$-Kirk-Saliga) mapping if $G_{\leqslant}$-monotone (resp., $G_{\leqslant}$-KirkSaliga) mapping.

An analogue version of Theorem 10 in the setting of the preordered metric spaces may be stated as follows.

Theorem 15. Let $(X, d, \leqslant)$ be a preordered complete metric space satisfying the (OSCL) condition. Let $T: X \rightarrow X$ be $a \leqslant-$ monotone and $\leqslant-K i r k$-Saliga mapping. If there exists an element $x_{0} \in X$ such that $T x_{0} \leqslant x_{0}$, then $T$ admits a fixed point in $X$.

Remark 16. If moreover the above binary relation $\leqslant$ is antisymmetric (i.e., $(x \leqslant y$ and $y \leqslant x$ ) imply $x=y$ ), we obtain, from Remark 6, the following result established by Alfuraidan and Khamsi.

Corollary 17 (see $[16$, Theorem 5$]$ ). Let $(X, d, \leqslant)$ be a complete partially ordered metric space satisfying the property (OSC). Let $T: X \rightarrow X$ be a $\leqslant$-monotone and $\leqslant-$ Kirk-Saliga mapping with $p=1$. If there exists an element $x_{0} \in X$ such that $T x_{0} \leqslant x_{0}$, then $T$ admits a fixed point in $X$.

\section{Conflicts of Interest}

The authors declare that they have no conflicts of interest.

\section{References}

[1] B. Lemmens and R. Nussbaum, Nonlinear Perron-Frobenius theory, vol. 189 of Cambridge Tracts in Mathematics, Cambridge University Press, Cambridge, UK, 2012.

[2] S. Banach, "Sur les opérations dans les ensembles abstraits et leur application aux équations intégrales," Fundamenta Mathematicae, vol. 3, pp. 133-181, 1922.

[3] G. Teschl, Ordinary Differential Equations and Dynamical Systems, American Mathematical Society, Providence, RI, USA, 2012.

[4] M. Edelstein, "An extension of Banach's contraction principle," Proceedings of the American Mathematical Society, vol. 12, pp. 7-10, 1961.

[5] J. Caristi, "Fixed point theorems for mappings satisfying inwardness conditions," Transactions of the American Mathematical Society, vol. 215, pp. 241-251, 1976.

[6] H. Brézis and F. E. Browder, "A general principle on ordered sets in nonlinear functional analysis," Advances in Mathematics, vol. 21, no. 3, pp. 355-364, 1976.

[7] A. Brøndsted, "On a lemma of Bishop and Phelps," Pacific Journal of Mathematics, vol. 55, no. 2, pp. 335-341, 1974. 
[8] I. Ekeland, "On the variational principle," Journal of Mathematical Analysis and Applications, vol. 47, pp. 324-353, 1974.

[9] W. A. Kirk, "Caristi's fixed point theorem and metric convexity," Colloquium Mathematicum, vol. 36, no. 1, pp. 81-86, 1976.

[10] W. A. Kirk and L. M. Saliga, "The Brézis-Browder order principle and extensions of Caristi's theorem," Nonlinear Analysis: Theory, Methods \& Applications, vol. 47, no. 4, pp. 2765-2778, 2001.

[11] M. Turinici, "Extension of the Kirk-Saliga fixed point theorem," Analele stiintifice ale Universitatii Ovidius Constanta, vol. 15, no. 2, pp. 91-102, 2007.

[12] A. C. Ran and M. C. Reurings, "A fixed point theorem in partially ordered sets and some applications to matrix equations," Proceedings of the American Mathematical Society, vol. 132, no. 5, pp. 1435-1443, 2004.

[13] J. J. Nieto and R. Rodríguez-López, "Contractive mapping theorems in partially ordered sets and applications to ordinary differential equations," Order, vol. 22, no. 3, pp. 223-239, 2005.

[14] R. P. Agarwal, M. A. El-Gebeily, and D. O’Regan, "Generalized contractions in partially ordered metric spaces," Applicable Analysis: An International Journal, vol. 87, no. 1, pp. 109-116, 2008.

[15] J. J. Nieto and R. Rodríguez-López, "Existence and uniqueness of fixed point in partially ordered sets and applications to ordinary differential equations," Acta Mathematica Sinica, vol. 23, no. 12, pp. 2205-2212, 2007.

[16] M. R. Alfuraidan and M. A. Khamsi, "Caristi fixed point theorem in metric spaces with a graph," Abstract and Applied Analysis, vol. 2014, Article ID 303484, 5 pages, 2014.

[17] M. Rigo, "Advanced Graph Theory and Combinatorics," John Wiley \& Sons, 2016.

[18] M. A. Khamsi and W. A. Kirk, An Introdunction toMetric Spaces and Fixed Point Theory, Wiley, New York, NY, USA, 2001. 


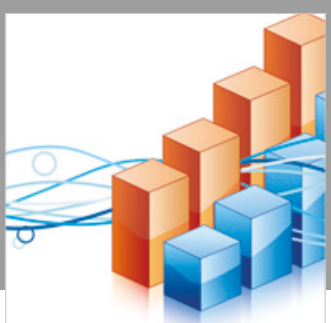

Advances in

Operations Research

\section{-n-m}
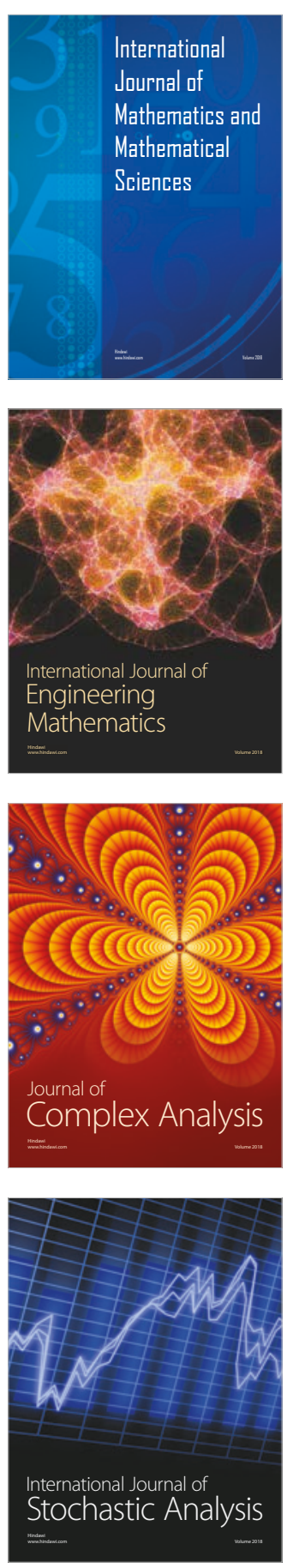
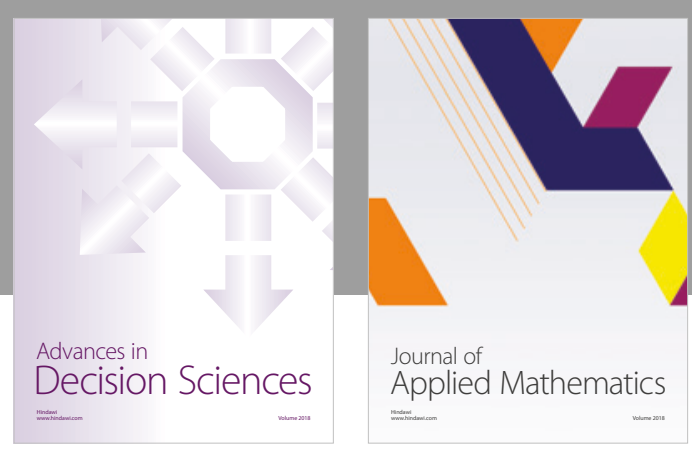

Journal of

Applied Mathematics
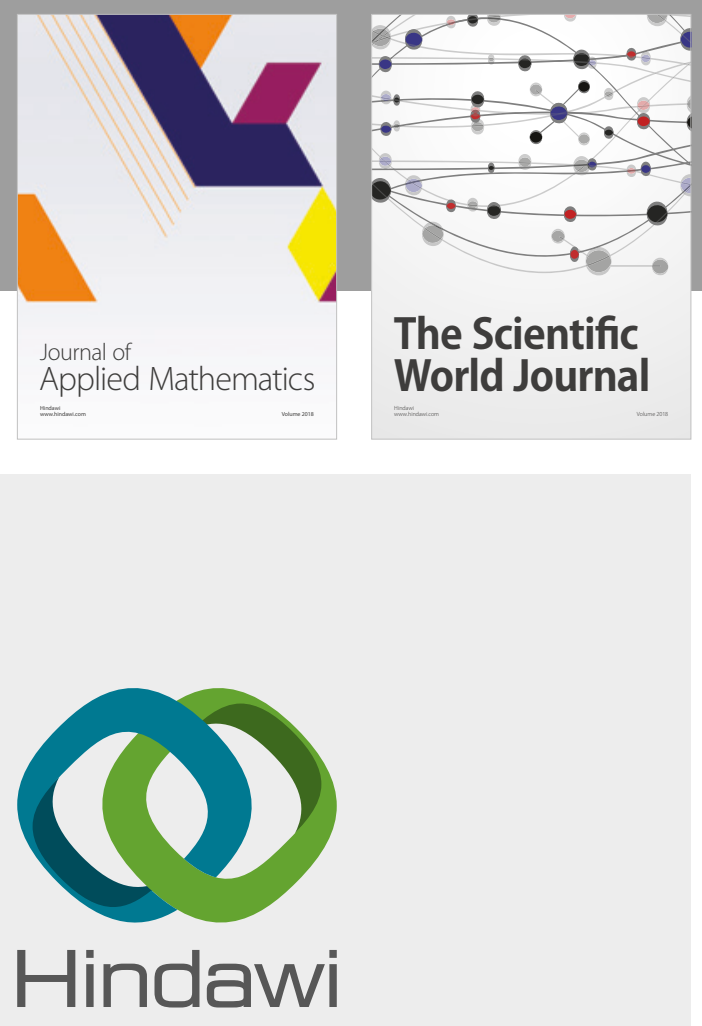

Submit your manuscripts at

www.hindawi.com

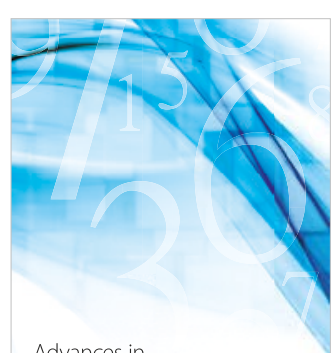

Advances in
Numerical Analysis
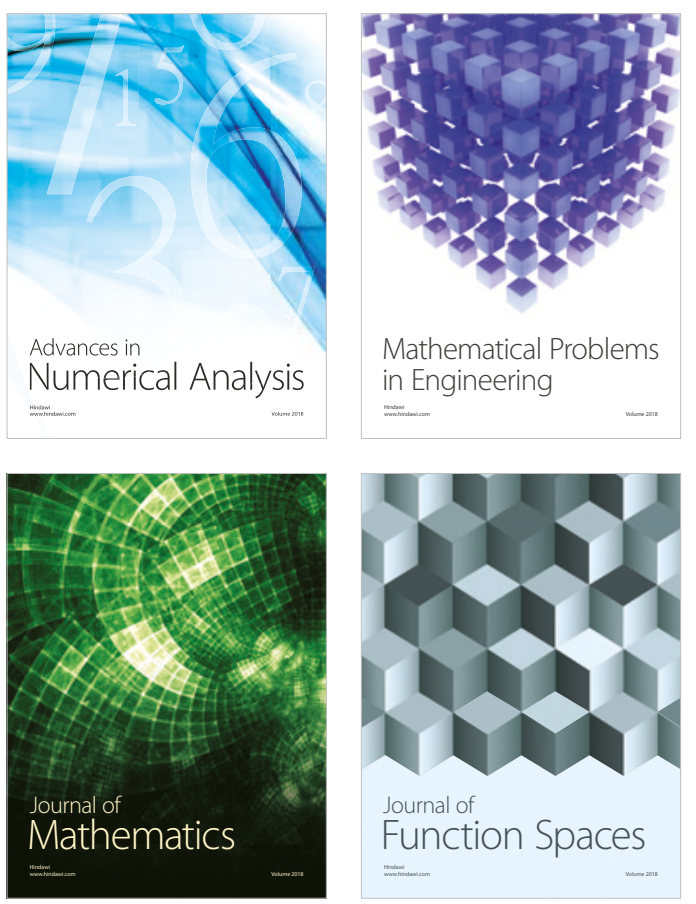

Mathematical Problems in Engineering

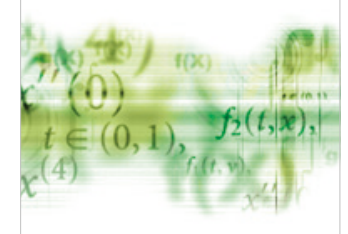

International Journal of

Differential Equations

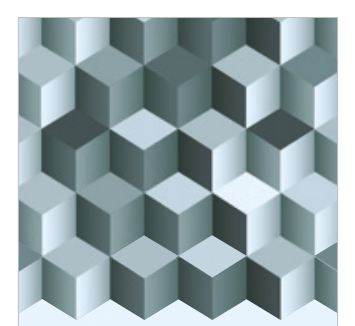

Journal of

Function Spaces

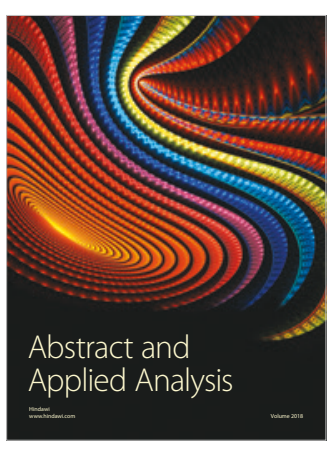

The Scientific

World Journal

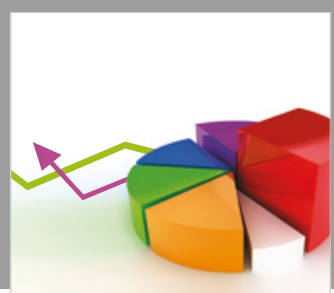

Journal of

Probability and Statistics
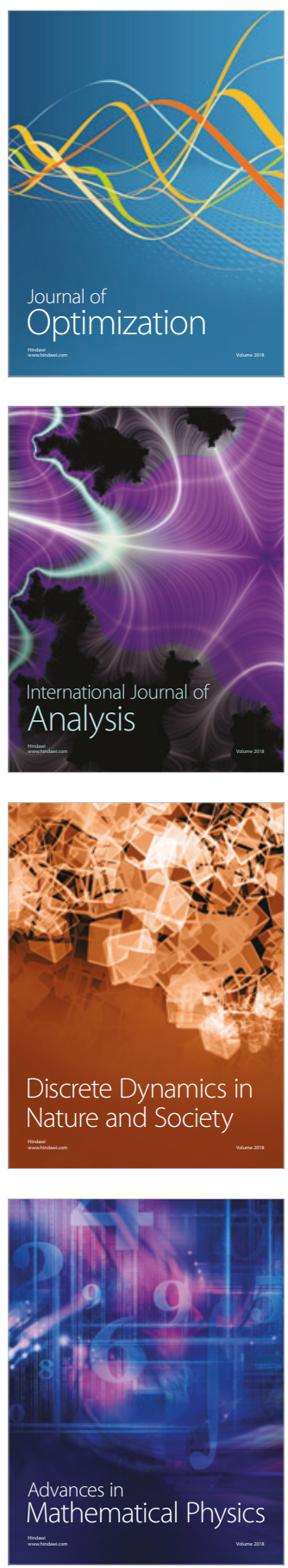